\title{
Experimental research of Fuaile medical adhesive for portal vein embolization in white rabbit models
}

\author{
TIANPENG JIANG, LIZHOU WANG, XING LI, JIE SONG, XIAOPING WU, TIANZHI AN and SHI ZHOU \\ Department of Radiology, Affiliated Hospital of Guiyang Medical College, Guiyang, Guizhou 550004, P.R. China
}

Received July 11, 2014; Accepted February 23, 2015

DOI: $10.3892 / 01.2015 .3142$

\begin{abstract}
The aim of the present study was to investigate the feasibility and efficacy of Fuaile medical adhesive for portal vein embolization in the treatment of a rabbit model. This study used 26 white rabbits, 14 of which were selected and assigned into seven groups $(n=2)$ for the preliminary experiment. Fuaile medical adhesive was mixed with lipiodol at different ratios of 1:0, 1:1, 1:2, 1:3, 1:4, 1:5 and 0:1, respectively, and administered via the portal trunk. The remaining 12 white rabbits were randomly divided into two groups $(n=6)$. The evaluation of the results included the degree of adhesion to the vessels, the extent of embolization and the reaction of the rabbit. Hepatic and renal functions were detected prior to and at 1, 7 and 14 days post-embolization, respectively. Angiography, CT scans and pathological examinations were conducted at post-embolization. Histological examinations revealed that the topical swollen lesions were darker. Light microscopy showed embolic agents in the portal venous blood vessels and the formation of a secondary thrombus. Hepatic necrosis appeared surrounding the embolization area. Inflammatory cell infiltration of different degrees occurred in the early stage and inflammatory fibroplasia occurred in the late stage. Alanine aminotransferase and aspartate aminotransferase levels increased at 1 day post-embolization, peaked at 7 days and was in the normal range at 14 days. The levels of blood urea nitrogen and ceruloplasmin were elevated at 1 day post-embolization and lowered to normal at 7 days. Fuaile medical adhesive is an effective, safe and inexpensive agent, used for effectively inducing embolization in the portal trunk, and the first and second branches of rabbit portal veins. The use of Fuaile therefore merits widespread application in clinical practice.
\end{abstract}

\section{Introduction}

Liver cancer is one of the common malignant tumors worldwide. Currently, surgery is the most effective treatment for liver

Correspondence to: Dr Shi Zhou, Department of Radiology, Affiliated Hospital of Guiyang Medical College, 28 Guiyi Road, Guiyang, Guizhou 550004, P.R. China

E-mail: shizhou123@yeab.net

Key words: portal vein embolization, Fuaile medical adhesive cancer. However, some patients may not be suitable for surgical treatment due to postoperative complications including liver failure caused by small future liver remnants (FLRs). Portal vein embolization (PVE) is a procedure that induces regrowth on one side of the liver prior to planned hepatic resection of the other side. The procedure greatly reduces the risk of postoperative liver failure by increasing FLR, and may enable patients whose tumors were previously deemed inoperable due to small FLR, eligible for surgical treatment (1). Portal vein embolization (PVE) is used pre-operatively for patients with primary or metastatic liver cancer, or as a palliative treatment combined with chemotherapy for non-surgical cases. Embolic materials are the key factor determining the success of PVE. At present, common embolic agents for portal veins include absolute ethanol, gelatin sponge particles, spring rings and Bletilla striata. Absolute ethanol mainly affects the secondary embolization of peripheral vessels and the great arteries $(2,3)$, and is a type of vascular embolic agent, as well as a tissue necrotic agent. However, it is likely to cause regurgitation and the dosage is difficult to control, thereby inducing inadvertent occlusion of the wrong vessels. Alternative liquid embolic agents are likely to adhere to the blood vessels, leading to vascular injury, multiple complications, a high risk of vascular complications, vascular toxicities and even malignant tumors. The use of Ony ${ }^{\circledR}$ requires a specific, expensive catheter. These limitations prevent the clinical application of the agents.

Fuaile medical adhesive is the third generation of medical adhesive invented by Chinese researchers. Fuaile is characterized as being highly adhesive with good diffusion properties; it is a low polymerization heat adhesive with a mild bleaching phenomenon and desirable polymer toughness. Moreover, Fuaile medical adhesive exerts a stable effect without causing side-effects, toxicity, abnormalities or carcinoma $(4,5)$. When Fuaile medical adhesive is diluted with lipiodol, an inexpensive agent, at varying ratios, the price of medical treatment may decrease. Fuaile possesses the preliminary requirements for use as a vascular embolic agent, including being stable, non-toxic, non-teratogenic and non-carcinogenic. In China, experimental studies have confirmed the successful use of Fuaile medical adhesive in the embolization of the canine mesentery and branch vessels of the gastroepiploic veins. However, there have been no studies to verify that the application in the portal vein causes embolization.

In the present study, Fuaile medical adhesive mixed with lipiodol ultra-fluid at various ratios was used as a liquid 
Table I. The reactions of the portal trunk following infusion with embolic agent containing varying ratios of medical adhesive and lipiodol.

\begin{tabular}{lllll}
\hline Group & Degree of adhesion to microcatheter & Distribution area & $\begin{array}{c}\text { Vascular } \\
\text { recanalization }\end{array}$ & Rabbit systemic reactions \\
\hline $\begin{array}{l}\text { Pure medical adhesive } \\
1: 1\end{array}$ & Tightly adheres to catheter & Main trunk & None & Death \\
$1: 2$ & Prone to tightly adhere to catheter & Main trunk & None & Serious, prone to die \\
$1: 3$ & Adheres to catheter, but not tightly & Main trunk, first branch & None & Moderately serious \\
$1: 4$ & Partially adheres to catheter & First-second branches & None & Moderate to significant \\
$1: 5$ & Not prone to adhesion to catheter & First-second branches & None & Mild to moderate \\
Pure lipiodol & Not prone to adhesion to catheter & Second branch and below & None & Relatively mild \\
& Does not adhere to catheter & Second branch and below & None & Extremely slight \\
\hline
\end{tabular}

embolic agent for the selective embolization of the main trunk or branch vessels of the portal vein. This experiment aimed to investigate the feasibility and efficacy of applying Fuaile medical adhesive for rabbit PVE, providing experimental evidence for its subsequent clinical application.

\section{Materials and methods}

Experimental animals. A total of 22 healthy white rabbits, similar in age and weight, both male and female, were selected for the present study (Laboratory Animal Center, Guiyang Medical College, Guiyang, China). This study, inclusive of all experimental procedures involving animals, was approved by the Ethical Committee of the Affiliated Hospital of Guiyang Medical College.

Preparation of experimental materials. The study equipment consisted of a modified 18-gauge puncture needle, a $2.7 \mathrm{~F}$ concentric microcatheter system (microcatheter and microlead) and 1-, 5- and 10-ml syringes. Additionally, an Axiom Artis angiography instrument (Axiom Artis dFC; Siemens Medical Solutions, Erlangen, Germany), GE HiSpeed CT/i scanner (GE Medical Systems, Milwaukee, WI, USA), Lecia RM 2025 microtome (Lecia Instruments Ltd, , Nussloch, Germany), HB-2 optical microscopy and imaging system (Olympus, Tokyo, Japan), MPIAS-9000 multimedia true-color pathology image analysis system (Tongji University, Shanghai, China) were employed.

The agents used were as follows: Fuaile medical adhesive was evenly daubed at the indicated sites (specification, $0.5 \mathrm{ml} / \mathrm{U}$; batch number, 20070829; Beijing Fuaile Science And Technology Development Co., Ltd., Beijing, China); lipiodol ultra-fluid injection (specification, $10 \mathrm{ml}$; batch number, 06LU006A; Guerbet, Villepinte, France); iopromide injection (Uhravist 370; specification, $100 \mathrm{ml}, 37 \mathrm{~g} / \mathrm{l}$; batch number, 1000367; Bayer Healthcare Co., Ltd., Guangzhou, China); ketamine hydrochloride injection (specification, $2 \mathrm{ml}$, $0.1 \mathrm{~g}$; batch number, KH070401; Jiangsu Hengrui Medicine Co., Ltd., Lianyungang, China); and heparin sodium injection (specification $2 \mathrm{ml}, 12,500$ units; Tianjin Biochem Pharmaceutical Co., Ltd., Tianjin, China).

Experimental procedure. Prior to embolization, under anesthesia, a blood sample was collected from the rabbits via the auricular veins in order to perform hepatic and renal function tests. These tests were performed again at 1,7 and 14 days post-operatively.

First, preliminary experiment. A total of 14 white rabbits were randomly divided into 7 groups, with 2 rabbits in each group. Medical adhesive and lipiodol ultra-fluid were mixed at a ratio of 1:0, 1:1, 1:2, 1:3, 1:4, 1:5 (adhesive groups) and 0:1 (lipiodol group). The microcatheter head was placed at the portal trunk and the injection was gradually delivered at $0.01 \mathrm{ml} / \mathrm{s}$ for a total volume of $0.2 \mathrm{ml}$. The approximate adhesion time, degree of adhesion and range of distribution were observed.

Second, formal experiment. A total of 12 white rabbits were collected and randomly assigned into 2 groups, with 6 rabbits in each group. The first and second blood vessel branches were used as the target vessels. Based upon the preliminary experiment data, medical adhesive was mixed with lipiodol ultra-fluid at a ratio of 1:4 and used at the same speed. Blood vessel embolization was performed in each group.

\section{Experimental methods}

Preoperative preparation. All animals were subject to fasting pre-operatively, fixed on the operative bench in a supine position and underwent anesthesia using $1 \mathrm{ml}$ ketamine plus $5 \mathrm{ml}$ normal saline via the auricular veins. The animals also received persistent intravenous administration of $2 \mathrm{ml}$ ketamine plus $150 \mathrm{ml}$ $5 \%$ glucose in water (GS) $(6,7)$. The hairs on the abdomen were shaved and conventional sterilization was performed.

Arterial intubation. Following repeated disinfection, an incision was made in the skin of the abdomen, exposing the portal trunk, and a puncture needle was used for portal trunk puncture. A microcatheter was introduced into the portal trunk for CT portography and then intubation was performed into the portal vein branches, guided by the microcatheter lead.

Embolization. The mixture of Fuaile medical adhesive and lipiodol ultra-fluid was injected at a constant speed following proper intubation, to avoid the incidence of embolic agent regurgitation. Following embolization, 5\% dextrose solution was used for irrigating the catheter, the microcatheter was withdrawn to the portal trunk and portal vein angiography was repeatedly performed to confirm the effect of embolization.

Observations. In each group, two animals were sacrificed at 1, 7 and 14 days post-angiography, and CT scan results and the liver tissues were collected for subsequent observation. The tissues were fixed in $10 \%$ formalin, and stained with 

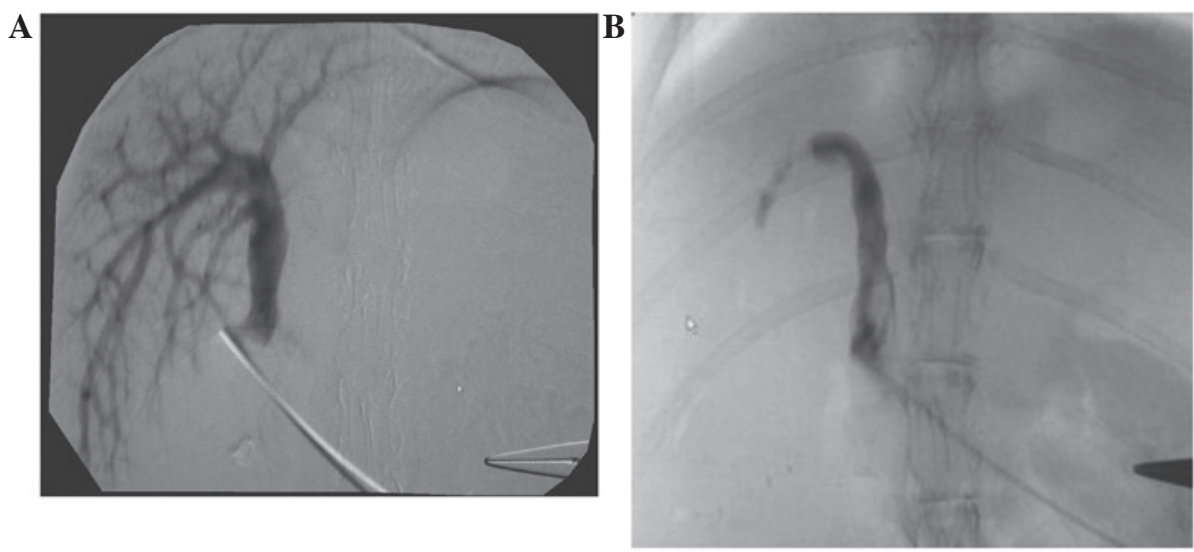

Figure 1. Embolization was formed by injection of embolic agents (1:4) through a microcatheter head placed at the portal trunk. (A) Substraction angiography of the portal vein. (B) Excessive embolization in the second branch of the portal vein induces acute complete embolization in the portal trunk. Embolic agents are shown as a high-density shadow.
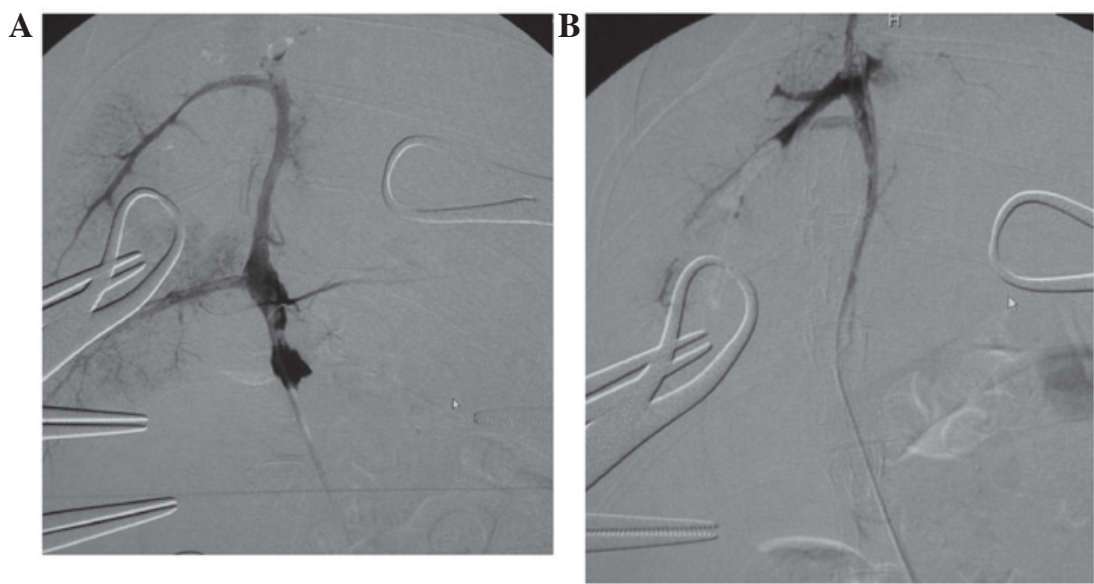

Figure 2. Embolization was formed by injection of embolic agents (1:4) through a microcatheter head placed at the proximal end of the second branch of the portal vein. (A) Substraction angiography of the portal vein. (B) Substraction angiography of the second branch of the portal vein post-embolization. The shadows of the second branch of the portal veins and its branches did not develop.

hematoxylin and eosin to observe the liver cell necrosis and PVE. Prior to embolization, blood samples were collected via the auricular veins at 1,7 and 14 days post-operatively to measure the liver and renal functions.

Statistical analysis. SPSS 11.5 statistical software (SPSS, Inc., Chicago, IL, USA) was used for the data analysis. All data are presented as the mean \pm standard deviation. A paired t-test was conducted and $\mathrm{P}<0.05$ was considered to indicate a statistically significant difference.

\section{Results}

Preliminary experimental results. A total dose of $0.01 \mathrm{ml}$ of embolic agent was infused into the portal trunk at varying ratios (Table I). The high doses of embolic agents (1:1 and 1:2) were much more likely to adhere to the catheter compared with the low doses (1:4 and 1:5). While high doses of embolic agents were distributed in the main trunk and first branch, the low doses were localized to the first-second and second branches. Furthermore, rabbits in the high-dose groups had a stronger systemic reaction to the embolic agents compared with the low-dose groups.
Dose of embolic agents. The injected doses of embolic agents were determined according to rabbit size and the speed of blood flow. The doses were $\sim 0.15 \mathrm{ml}$ for the portal trunk, and $0.02 \mathrm{ml}$ for the first branch and $0.01 \mathrm{ml}$ for the second branch of the portal veins.

Speed of embolization. The overall speed was extremely slow and maintained under strict supervision. The high doses (1:1 and 1:2) more commonly induced regurgitation and embolized the blood vessels in error due to heavy resistance, while the low doses of embolic agents (1:4 and 1:5) less commonly caused the incidence of regurgitation, and was easy to manage.

Formal experimental results. Based on the preliminary experimental outcomes, embolization was found to be optimal at a 1:4 ratio. In consideration of the severe reactions, including death, following portal trunk embolization, the first or second branches of the portal vein were also selected for embolization. The dose of embolic agents for the first branch of the portal vein was $0.02 \mathrm{ml}$ and the dose for the second branch was $0.01 \mathrm{ml}$. A total of 6 rabbits were subjected to embolization of the first branch of the portal vein and 6 were 

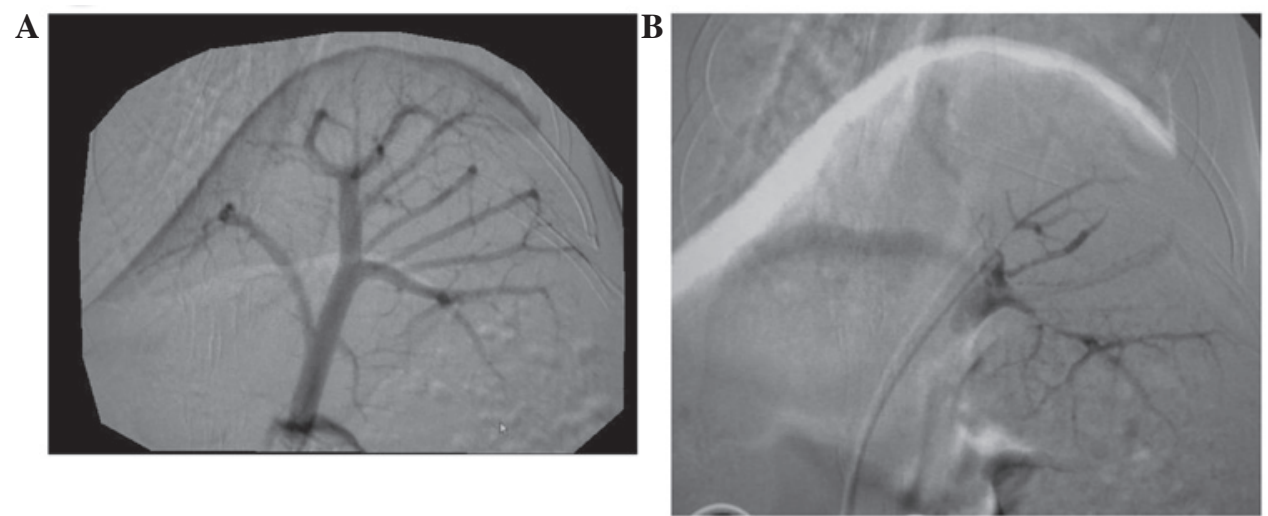

Figure 3. Embolization was formed by injection of embolic agents (1:4) through a microcatheter head placed at the first branch of the portal vein. (A) Substraction angiography of the portal vein. (B) Substraction angiography of the first branch of the portal vein post-embolization. The shadows of the first branch of the portal vein partially developed due to incomplete embolization.

A
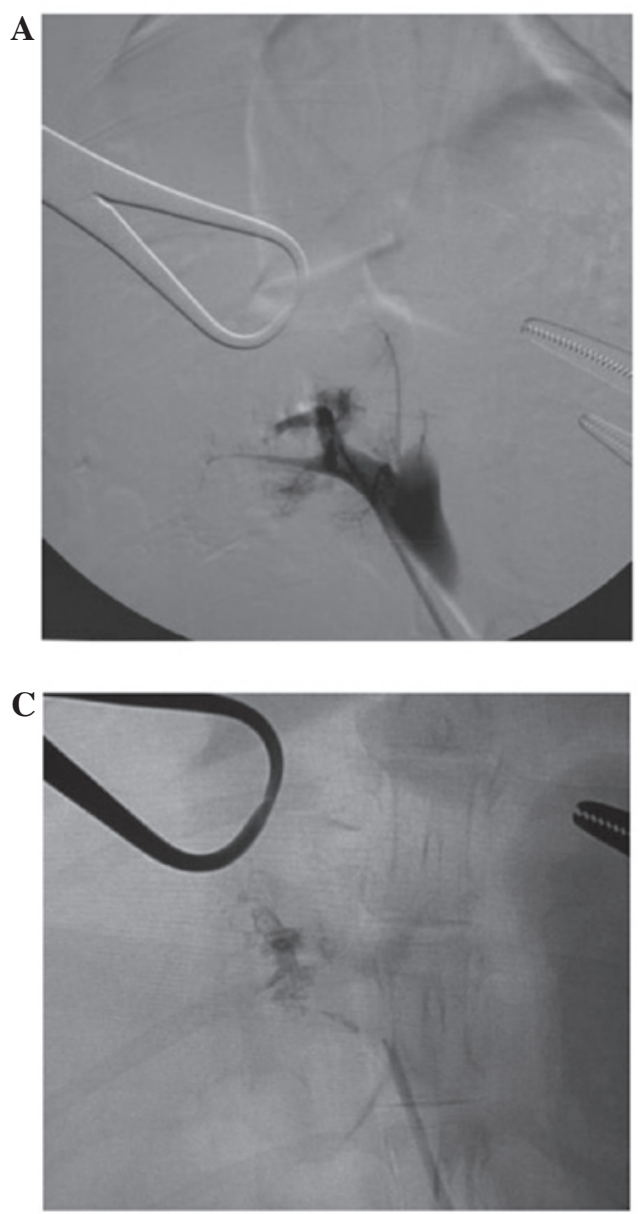

B
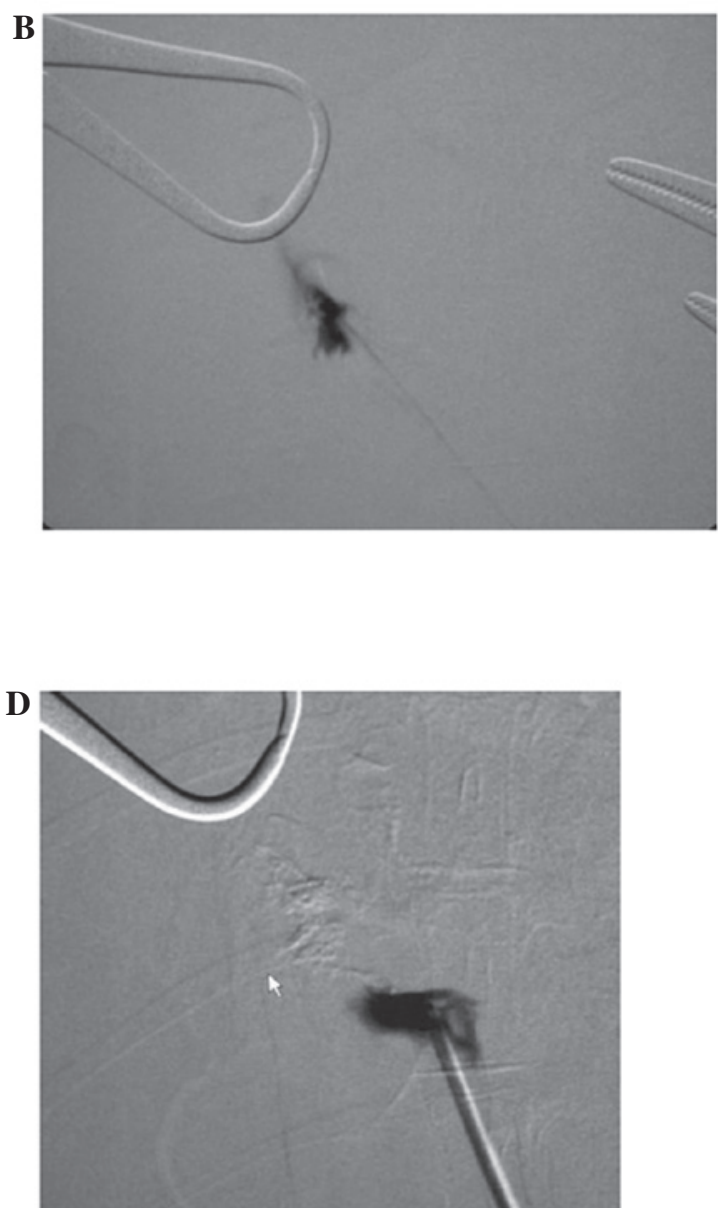

Figure 4. Embolization was formed by injection of embolic agents (1:4) through a microcatheter head placed at the second branch of the portal vein. (A) Substraction angiography of the portal vein. The head of the microcatheter was located in the second branch of the portal vein. (B) Substraction angiography of the second branch of the portal vein. (C) High-density shadows of the embolic agents were observed following the embolization of the second branch of the portal vein. (D) Substraction angiography of the second branch of the portal vein post-embolization. Complete embolization was apparent in the target blood vessels and its branches, and regurgitation of the contrast agent was noted.

subjected to embolization of the second branch, with a success rate of $100 \%$.

CT portography. All cases received an acute and complete embolization. The embolization rate achieved was $100 \%$. With the exception of the pure lipiodol group, no other groups experienced significant recanalization at 7 and 14 days post-embolization (Figs. 1-4).

CT scanning. Conventional CT scanning revealed high-density shadows in the targeted blood vessels, but the density of the liver tissues did not decrease significantly. Post-embolization 
Table II. Comparison of hepatic and renal function before and 1, 7, and 14 days after embolization of portal vein.

\begin{tabular}{|c|c|c|c|c|}
\hline \multirow[b]{2}{*}{ Hepatic function } & \multirow[b]{2}{*}{ Prior to embolization } & \multicolumn{3}{|c|}{ Post-embolization } \\
\hline & & 1 day & 7 days & 14 days \\
\hline ALT, U/1 & $35.10 \pm 1.10$ & $64.30 \pm 7.60$ & $295.50 \pm 19.40^{\mathrm{a}}$ & $37.29 \pm 1.56$ \\
\hline AST, U/1 & $46.30 \pm 2.40$ & $67.60 \pm 6.30$ & $182.70 \pm 10.60^{\mathrm{a}}$ & $47.45 \pm 1.98$ \\
\hline BUN, mmol/l & $8.00 \pm 1.46$ & $10.25 \pm 1.97$ & $8.52 \pm 1.85$ & $8.37 \pm 1.65$ \\
\hline $\mathrm{Cr}, \mu \mathrm{mol} / 1$ & $94.50 \pm 2.14$ & $101.36 \pm 2.86$ & $97.28 \pm 2.36$ & $95.32 \pm 2.25$ \\
\hline
\end{tabular}

${ }^{\mathrm{a}} \mathrm{P}<0.05$. ALT, alanine aminotransferase; AST, aspartate aminotransferase; BUN, blood urea nitrogen; CR, ceruloplasmin.
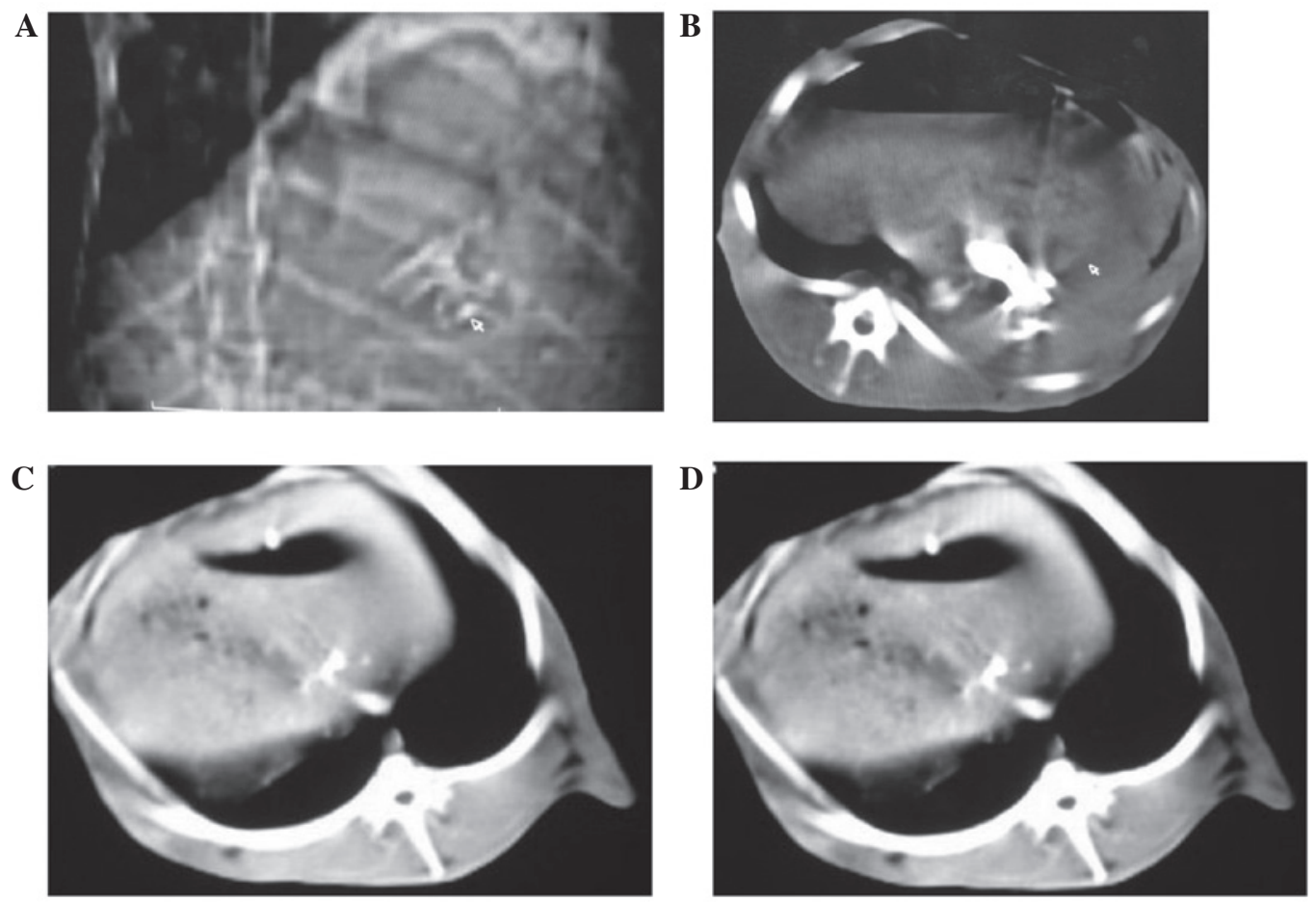

Figure 5. (A) Computed tomography (CT) scans displaying high-density shadows of the embolic agents in the liver tissues. (B) CT scan at the soft-tissue window following the embolization of the portal trunk and the first branch of the portal vein; embolic agents are present as high-density shadows. (C) At 7 days post-embolization of the first branch of the portal vein, high-density shadows of the embolic agents were apparent in the corresponding blood vessels. The density of the liver tissue in the blood supply area was decreased and disorderly spot gas shadows were noted. (D) CT scan at the soft-tissue window at 14 days post-embolization of the first branch of the portal vein showed that the high-density shadows of the embolic agents remained in the corresponding blood vessels. The density of the liver tissue in the blood supply area was more significantly decreased and the number of spot gas shadows was slightly increased.

reexamination identified the presence of high-density shadows. However, the density of the hepatic tissues was significantly decreased and a gas density shadow was visible (Fig. 5).

Histological examinations. To the naked eye, no significant changes occurred during the early stages post-embolization, and then topical swollen tissues gradually became darker. Upon light microscopy, during the early stage, the blended embolus of Fuaile medical adhesive and lipiodol was observed in the portal vein and partial central vein, followed by secondary formation of a thrombus, liver tissue necrosis within the embolization area, liver cell edema and cytoplasmic relaxation. The inflammatory response surrounding the embolization area was not significant during the early stage; inflammatory cell infiltration of varying degrees was evident and apparent inflammatory fibroplasia occurred 14 days later. A substantial amount of lipiodol and medical adhesive mixture was observed followed by secondary formation of a thrombus. No significant vascular recanalization was recorded (Fig. 6).

Changes in hepatic and renal functions before and after embolization (Table II). As shown in Table II, the liver function damage occurred at 1 day post-embolization, prior to the severity peaking at 7 days and subsequently being almost restored to normal status at 14 days, as indicated by levels of alanine aminotransferase (ALT) and aspartate aminotransferase (AST). Renal function damage was identified at 1 day post-embolization, and normal function resumed at 7 days, as indicated by levels of blood urea nitrogen (BUN) and ceruloplasmin (CR). 
$\mathbf{A}$
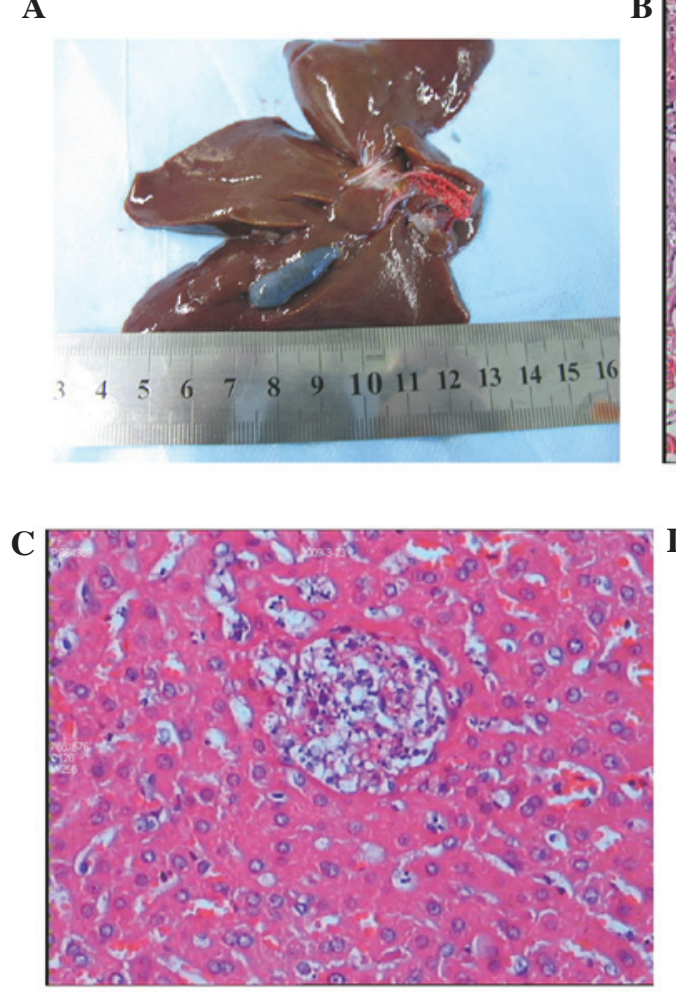
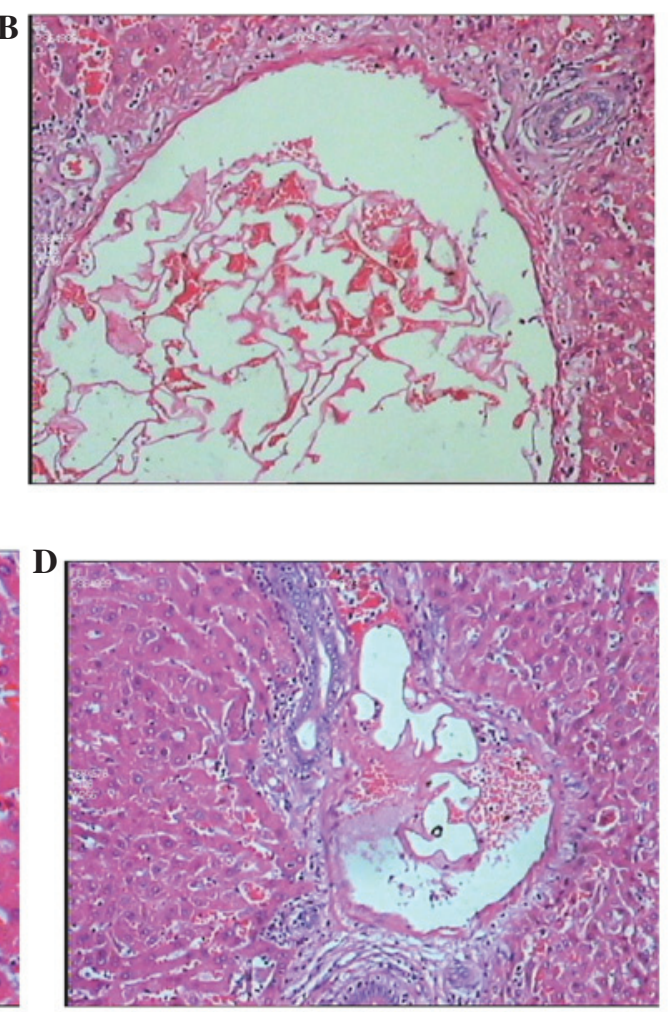

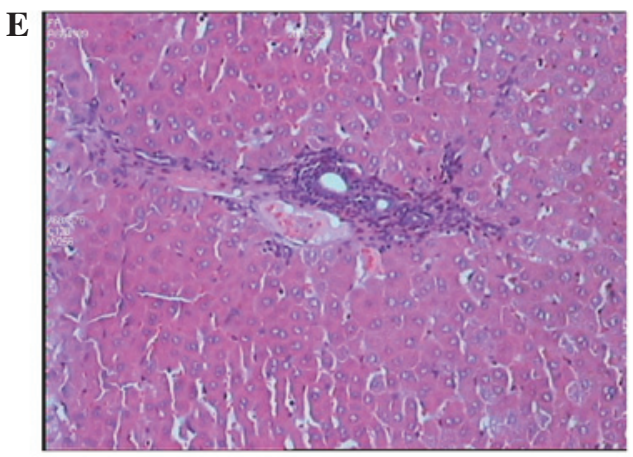

Figure 6. (A) Following portal trunk embolization, red embolic agents and secondary formation of a thrombus were noted in the portal trunk. (B) Embolization of the largest branch of the portal vein (magnification, x100). A large amount of blended lipiodol and Fuaile medical adhesive was observed as an embolus, followed by secondary formation of a thrombus. (C) At 1 day post-embolization, liver cell injuries were observed, typical necrotic lesions formed and inflammatory cell infiltration was insignificant (magnication, x200). (D) At 7 days post-embolization of the portal vein branches, an embolus and secondary formation of a thrombus were noted. The inflammatory cell infiltration was more serious surrounding the embolized blood vessels (HE staining; magnification, x100). (E) At 14 days post-embolization, inflammatory hyperplasia was apparent in the embolized area, and adjacent liver cell necrosis was also noted (HE staining, magnification, x100). HE, hematoxylin and eosin.

\section{Discussion}

Transhepatic portal catheterization for PVE is effective in pre-operative embolization for patients with primary or metastatic liver cancer, or as a palliative treatment combined with chemotherapy for non-surgical cases (8-12). For patients receiving pre-operative embolization, embolization of the branch of the partial portal vein can cause the atrophy of liver tissue lesions, reduced tumor size and lead to hepatic tissue hyperplasia within the non-embolization area, thereby widening the extent of the surgical excision and surgical indications.

Furthermore, PVE also avoids the acute liver cell damage induced by a decline in portal venous pressure intraoperatively or abnormal metabolism resulting from excessive hyperplasia post-operatively, which enhances the tolerability of surgery and decreases the incidence of post-operative complications $(13,14)$. For non-surgical patients, large peripheral lesions of primary liver cancer supply blood for the portal vein, while metastatic liver cancer depends on the portal vein for its blood supply. Liver artery chemotherapy combined with embolization yields poor clinical effects, with a high incidence of metastasis and recurrence post-operatively $(12,13)$. Consequently, performing combined chemotherapy and embolization therapy in the liver artery and portal vein of patients with liver cancer not only thoroughly treats intrahepatic malignant masses, but also reduces the incidence of tumor recurrence and metastasis. In addition, it is also capable of treating portal venous tumor thrombosis.

Fuaile medical adhesive was initially invented by using $N$-octyl- $\alpha$-cyanoacrylate (NOCA) to alter the property of 
$N$-butyl cyanoacrylate (NBCA). In total, $>99 \%$ of its ingredients are $\alpha$-cyano methoxyethyl acrylate, NOCA and NBCA. Xia et al (4) adapted the formula of Fuaile in 2003 to create a spray-adehesive. Fuaile medical adhesive is categorized as a 6865-III medical device, which is widely applied in intraoperative hemostasis, adhesion, sealing and embolization. Thus far, 17 biological detections and >100 pathological tests, including asepsis, pyrogen, acute systemic toxicity, sub-acute poisoning, skin sensitization, intracutaneous irritation, hemolysis, cell toxicity and the Ames test, have been completed and yielded negative results. The experimental outcomes for mutagenic, teratogenic and carcinogenic effects, and propagation were also negative. Antibacterial tests revealed that the adhesive exerts an inhibitory effect on 11 species of bacteria, including Staphylococcus aureus. No adverse reactions have been reported in the 29-year follow-up of $>10$ million cases in clinical settings. Pathological studies conducted in the Second Affiliated Hospital of Xi'an Medical University in order to utilize Fuaile medical adhesive to embolize the canine mesentery and branch vessels of the gastroepiploic vein, noted aseptic inflammatory reactions at days $1-4$, which were alleviated at 7 days and significantly eased at 14 days. Intravenous membrane hyperplasia invaded the vascular lumen and surrounded the medical adhesive, splitting it into different partitions. At 14 days, fibrosis appeared in the vascular wall and fibrous embolization was observed in the venular lumen. Residual medical adhesive embraced by fiber tissues remained in the large venous lumen for up to one year, and was classified as permanent embolization.

NOCA and NBCA are useful as blood vessel embolic agents in a clinical setting. However, they exhibit significant adhesion and there is a risk of the microcatheter adhering to the blood vessels. Additionally, aggregation effects may give off heat. Fuaile medical adhesive precludes a variety of additives and is safe for human use. Compared with NOCA AND NBCA, Fuaile medical adhesive has an appropriate polymerization speed, low polymerization heat and desirable diffusion. The preliminary experiments of the present study found that if Fuaile medical adhesive was mixed with lipiodol ultra-fluid at a proper ratio and infused at proper speed, the incidence of adherence to the blood vessels was prevented and the goal of being able to perform repeat injections in the embolized vessels was met.

Compared with non-adhesive liquid embolic agents, such as Onyx, Fuaile medical adhesive has a lower price, yields no vascular toxicity and can be easily injected via common catheters. Moreover, Fuaile medical adhesive can be prepared as an embolic agent for different branches of target blood vessels, whereas absolute ethanol is limited to application in peripheral vessel embolization $(4,14)$. Therefore, Fuaile medical adhesive is feasible in clinical practice as a liquid embolic agent.

With regard to the safety of PVE, the hepatic and renal function tests performed in the present study indicated that liver function damage emerged at 1 day post-embolization, prior to the severity peaking at 7 days and then being almost restored to normal status at 14 days. Renal function damage appeared at 1 day post-embolization, and normal function resumed at 7 days. These results are consistent with previous findings. Dong et al conducted PVE in rat models and noted alanine aminotransferase (ALT) elevation at 1 day post-embolization, which started to decline at 7 days and was finally restored to a normal level at 14 days (15). Wan et al (16) utilized Bletilla microspheres and absolute ethanol to perform PVE in rabbits, and subsequent liver function examination indicated that liver function damage occurred at 1 day post-embolization, and that the severity of the injuries peaked at approximately 3 days and were generally restored to a normal level 14 days later. Wu et al (17) applied a type of medical adhesive, DTH for selective PVE in a rat model and found that liver function presented with transient changes characterized as ALT and AST elevation 1 day post-operatively, which started to decline at 3 days and eventually returned to normal levels 14 days post-operatively. Dong et al (15) used NBCA to perform PVE in rats. This caused an increase in the level of ALT at 1 day post-embolization. A decreasing trend was evident at 7 days. At 14 days, liver and renal functions appeared to be at normal levels. In this study, renal function injuries were relatively mild and rapidly recovered (15), however, the possibility of contrast agent-induced renal toxicity must always be considered.

Wan et al (16) suggested that PVE is generally safe if the extent of embolization does not exceed three liver segments. In this investigation, the animals showed a slightly poor performance with regard to diet, physical strength and activity at 1 day post-embolization, but all were subsequently restored to normal status (16).

The rabbits died during the preliminary experiment, which may be explained by the following reasons: i) Excessive anesthesia-related mortality; ii) intraperitoneal hemorrhage-related mortality due to the improper management of hemostasis; and iii) acute complete portal trunk embolization-related mortality due to an excessive dose of embolic agents. Therefore, overall, it is safe to perform PVE in rabbit models if the procedures, including anesthesia, hemostasis and extent of embolization, are controlled.

Since Fuaile medical adhesive experiences a transient transition from a hydrophilic to hydrophobic status in water at $37^{\circ} \mathrm{C}$, attention is required upon the injection of embolic agents. The presence of sediment from the embolic agents must be avoided in the microcatheter, as it could possibly block the microcatheter and affects the surgery. Based on the findings from the in vitro embolization experiment and preliminary study, the following procedures are necessary: i) Prior to injection, the microcatheter should be washed using a moderate amount of phosphate-buffered saline to lower the temperature of the wall and cavity of the microcatheter, thereby extending the time of phase transition of the polymer in the microcatheter.

ii) Prior to and following injection, 5\% GS irrigation should be used to wash the microcatheter cavity to prevent the formation of sediment from the embolic agents within the microcatheter or around the mouth, which may lead to catheter obstruction or make the microcatheter adhere to the blood vessel wall.

iii) The total dose of embolic agents should be under control, with $\sim 0.15 \mathrm{ml}$ for the portal trunk, $0.02 \mathrm{ml}$ for the first branch of portal vein and $\sim 0.01 \mathrm{ml}$ for the second branch of the portal vein in rabbits. An increase in dosage of embolic agents is necessary for multiple injections. The dose for each injection should not be excessively high to avoid the incidence of embolization of the surrounding blood vessels or adhesion to the microcatheter. 
iv) The speed of injection for different doses of embolic agents should vary accordingly. It is critical that the embolic agents be injected extremely slowly using a 1-ml syringe. Repeated injections will prevent the incidence of regurgitation and embolization of other blood vessels in error. Special attention is necessary when injecting a high dose of embolic agents.

v) The entire process of embolization should be monitored to avoid errors.

vi) Angiography is to follow immediately after embolization to prevent the incidence of incomplete embolization.

vii) Injury to the animals must be avoided as much as possible. An incision of 3-4 $\mathrm{cm}$ in size on the abdominal wall at the site of the portal vein can be enlarged as necessary. Pre-operative fasting reduces the volume of the stomach to minimize the risk of anesthesia and to expose the portal vein easily.

viii) The frequency of intraoperative portal vein puncture should be reduced as much as possible. The puncture, the microcatheter and the needle should be in the correct position to prevent damage to the portal vein blood vessel wall and to avoid repeated punctures, which aggravate the severity of the trauma and enhance the difficulty of hemostasis. Hemostasis is achievable by suppressing the puncture site for several minutes after pulling out the needle. A suitable quantity of Fuaile medical adhesive should be daubed on the puncture point as necessary.

ix) The surgery must be performed in aseptic conditions. In the abdominal wall, incision sutures are applied layer by layer, then wrapped by bandages to minimize the risk of infection. The use of antibiotics in the diet is acceptable as necessary.

Insights gained from the present study included the fact that lipiodol ultra-fluid not only dilutes the concentration of Fuaile medical adhesive, but also allows for close monitoring throughout the process of embolization. By comparing CT portography images prior to and following embolization, it was found that the higher the dose of Fuaile medical adhesive injected, the larger the size of the branches of the portal vein that could be embolized. However, a higher dosage enhances the incidence of adhesion to the microcatheter and regurgitation. The present experimental results confirmed that the effect of embolization in the blood vessels was favorable and no significant recanalization was noted. The ratio of lipiodol ultra-fluid and lipiodol ultra-fluid should be $<1: 3$, and a ratio ranging from $1: 4$ to $1: 5$ is highly recommended. Further dilution is likely to prolong the time of polymerization and provide more time for the surgery, however, an excessively low concentration may negatively affect the effect of embolization.

In summary, Fuaile medical adhesive is a safe and efficacious liquid embolic agent that may be used for PVE in white rabbit models. The total dose differs depending upon the embolization site $(0.15 \mathrm{ml}$ for the portal trunk, $0.02 \mathrm{ml}$ for the first branch of the portal vein and $0.01 \mathrm{ml}$ for the second branch of the portal vein). In addition, the adhesive is useful in the embolization of different branches of the portal vein using selective catheterization and by combination with lipiodol ultra-fluid at varying ratios (recommended ratio, 1:3-1:5). Fuaile medical adhesive is cheap and easy to prepare, and therefore warrants widespread application in the clinical setting.

\section{References}

1. Abulkhir A, Limongelli P, Healey AJ, et al: Preoperative portal vein embolization for major liver resection: a meta-analysis $\square \mathrm{J} \square$. Ann Surg 247: 49-57, 2008.

2. Jungreis CA: Skuff-base tumors: ethanol embolization of the cavernous carotid artery. Radiology 181: 741-743, 1991.

3. Sadato A, Numaguchi Y, Taki W, et al: Nonadhesive liquid embolic agent: role of its components in histologic changes in embolized arteries. Acad Radiol 5: 198-206, 1998.

4. Xia HS, Tian X and Lu YS: A new generation of spray-type Fuaile medical adhesive (fundamental research). J Clin Surg 11: 2003 (In Chinese).

5. Xia HS, Tian X and Lu YS: A new generation of spray-type Fuaile medical adhesive (clinical research). J Clin Surg 11: 2003 (In Chinese).

6. Zheng ZL, Pan JS, Ma LJ, et al: The comparison of different anesthesia approaches for vascular surgery operation in rabbits. Journal of Hebei Medical University 29: 384-387, 2008 (In Chinese).

7. Li YQ, Yang XL, Qin JQ, et al: Analysis of application of ketamine in anesthesia of experimental animals. Shanghai Laboratory Animal Science 21: 169-170, 2001 (In Chinese).

8. Mao G, Yu Z, Zhang Y, et al: Combined transcatheter arterial chemoembolization and beta-ultrasound guided portal vein embolization in the treatment of hepatocellular carcinoma. Zhonghua Zhong Liu Za Zhi 24: 391-393, 2002 (In Chinese).

9. Bartolozzi C, Lencioni R, Caramella D, et al: Treatment of large HCC: transcatheter arterial chemoembolization combined with percutaneous ethanol injection versus repeated transcatheter arterial chemoembolization. Radiology 197: 812-818, 1995.

10. Kamada K, Kitamoto M, Aikata H, et al: Combination of transcatheter arterial chemoembolization using cisplatin-lipiodol suspension and percutaneous ethanol injection for treatment of advanced small hepatocellular carcinoma. Am J Surg 184: 284-290, 2002.

11. Tanaka H, Hirohashi K, Kubo S, et al: Preoperative portal vein embolization improves prognosis after right hepatectomy for hepatocellular carcinoma in patients with impaired hepatic function. Br J Surg 87: 879-882, 2000.

12. Inaba S, Takada $\mathrm{T}, \mathrm{Amano} \mathrm{H}$, et al: Combination of preoperative embolization of the right portal vein and hepatic artery prior to major hepatectomy in high-risk patients: a preliminary report. Hepatogastroenterology 47: 1077-1081, 2000.

13. Liu H, Peng YH, Yang ZH, et al: preoperative portal vein embolization increases the excision rate of liver cancer surgery. Chinese Journal of General Surgery 9: 583, 2004 (In Chinese).

14. Ji W, Ma KS, Dong JH, et al: Role of preoperative selective portal vein embolization in two-stage hepatectomy for primary hepatocellular carcinoma. Chinese Journal of Hepatobiliary Surgery 348-350, 2003 (In Chinese).

15. Dong BW, Liang P, Luo YK, et al: Experimental studies of the portal vein puncturing embolization with NBCA in rats. Chinese Journal of Ultrasonography 10: 494-497, 2001.

16. Wan ZY, Feng GS, Liang HM, et al: Experimental portal vein embolization in rabbits: a comparison of Bletilla microsphere with absolute ethanol. J Clin Radiol 23: 908-912, 2004 (In Chinese).

17. Wu XF, Fan J, Lin ZY, et al: Experimental studies on selective portal vein embolization. Journal of Chinese Microcirculation 4: 218-220, 2000 